\title{
Reliability Analysis of 6-Component Lattice Load-sharing Markov Repairable System with Spatial Dependence
}

\author{
Liying Wang*, Yuran Tian, Zhaona Pei \\ Shijiazhuang Tiedao Institute, Shijiazhuang, 050043 CHINA
}

\begin{abstract}
This paper proposes a new model that generalizes the traditional Markov repairable system to the case of spatial dependence among components. The components of the system are identical and arranged in two lines and consist of a lattice. The performance of each component depends on its spatial "neighbours" and the number of failed components in other lines. Markov process is adopted to model the performance of the system. The state space and transition rate matrix corresponding to a 6-component lattice load-sharing system with spatial dependence are presented. Availability of the system is obtained via Markov theory and Laplace transform method. A numerical example is given to illustrate the results in this paper. The states of the system are partitioned into four state sets: security, degraded, warning, and failed. The probabilities of visiting to four state sets are also discussed in the numerical example. The work might provide a basis for the reliability analysis of load-sharing systems with interacting components that themselves be arranged in some two-dimensional spatial pattern.
\end{abstract}

Keywords: Availability; Spatial dependence; Load-sharing system; Lattice system; Markov repairable system; Probability of visiting to secure states; Probability of visiting to warning states

(Submitted on November 13, 2016; Revised on April 17, 2017; Accepted on April 24, 2017)

(C) 2017 Totem Publisher, Inc. All rights reserved.

\section{Introduction}

Load-sharing systems are common in the real-world. Electric generators sharing an electrical load in a power plant, CPUs in a multiprocessor computer system, cables in a suspension bridge and valves or pumps in a hydraulic system are examples of load-sharing systems (see Kuo and Zuo 2003). Many empirical research results have indicated that the workload strongly affects a component's failure rate and an increased load induces a higher failure rate (see Kapur and Lamberson 1977, Iyer and Rossetti 1986, Barros et al. 2003). In a load-sharing system, if a component fails, the same workload has to be shared by the remaining components, resulting in an increased load shared by each surviving component. So how to describe the loadsharing rules and the dependences among components is an interesting topic and a significant body of research has been done. Load-sharing systems with equal, local, monotone and other load-sharing rules have been proposed. Amari et al. 2008 and Jain and Gupta 2012 presented remarkable reviews on the research. Various types of dependencies such as redundant configurations, failure dependence, common cause failures, propagated failures with global or selective effect and so on, have been considered (see Wang et al. 2013, Yu et al. 2007, Levitin and Xing 2010, Maaroufi et al. 2013).

The spatial dependence proposed in this paper comes from the operating of the large intelligent air conditioning system. The system usually consists of several components and these components are arranged according to the shape of the operation space. That is, the spatial pattern among components might be a line, a circle, a lattice, a central component surrounded by a

* Corresponding author. Tel.: 0086-031187935502.

E-mail address: wly_sjz@126.com. 
ring of other components and so on. A component has four performance rates: low, medium, high, and warning. When a component fails, the temperature sensors of its "neighbours" (not all components) will detect the change and increase their performance rates automatically. Therefore, the performance of each component in the system depends on its spatial "neighbours". The spatial "neighbours" of a component is relative to the spatial pattern in which the components of the system are arranged. So, this kind of dependence is different from those in literatures.

Under the assumption that a component's performance depends on its two spatial "neighbours" (left and right), Wang and Si 2014 consider the availability of a circular system using Markov theory. While for a large intelligent air conditioning system with lattice structure, the performance of each component depends not only on its nearest spatial "neighbours" but also the performance situation of components in other lines, due to the air flow. To model the operating process of these systems, a 6component lattice repairable system with spatial dependence is proposed.

The paper is organized as follows. A 6-component lattice load-sharing Markov repairable system with spatial dependence is formulated in Section 2 and the states of systems are also given in this section. Section 3 concentrates on the state transition analysis of the system. Availability of the system is obtained in Section 4. A numerical example is provided in Section 5. Availability, probability of visiting to four state sets are discussed in this section. Finally, a conclusion is given.

\section{Notations}

\begin{tabular}{|l|l|}
\hline$i k$ & $k$ th $(k=1,2,3)$ component in line $i(i=1,2)$ \\
\hline$l$ & number of a component's "neighbours" that are in failed state $(0 \leq l \leq 3)$ \\
\hline$\lambda$ & number of components that are in failed state in another line \\
\hline$X(t)$ & failure rate of a component; \\
\hline$S$ & state of the system \\
\hline$\tilde{S}$ & state space of continuous-time Markov process associated with the system \\
\hline$\tilde{X}(t)$ & aggregated process associated with $X(t)$ \\
\hline$Q$ & transition rate matrix associated with $\tilde{X}(t)$ \\
\hline$A(t)$ & instantaneous availability of the system \\
\hline$\pi$ & stationary distributions of the system \\
\hline$A$ & asymptotic availability \\
\hline$\tilde{S}_{1}$ & secure state set \\
\hline$\tilde{S}_{2}$ & degraded state set \\
\hline$\tilde{S}_{3}$ & warning state set \\
\hline$\tilde{S}_{4}$ & failed state set \\
\hline
\end{tabular}

\section{System formulation and states}

\subsection{6-component lattice load-sharing Markov repairable system with spatial dependence}

Assumptions for the 6-component lattice load-sharing Markov repairable system with spatial dependence are stated below.

(1) A 6-component lattice system consists of 6 identical components which are arranged in two lines. Each line consists of 3 components and they are linearly ordered. The $k$ th $(k=1,2,3)$ component in line $i(i=1,2)$ is denoted by $i k$. The "neighbours" of component $i k$ are components $i(k-1), i(k+1)$ and $i^{\prime} k\left(i^{\prime} \in\{1,2\}, i^{\prime} \neq i\right)$.

Note that components at the ends have two "neighbours", one is in the same line, one is in another line.

(2) Each component has two kinds of states: functional and failed. They may adjust their operating rates according to the states of their "neighbours" and the number of failed components in another line. Let $l(0 \leq l \leq 3)$ denote the number of a component's "neighbours" that are in failed state, $m(0 \leq m \leq n)$ denote the number of components that 
are in failed state in another line. The life-time durations of the components are exponential random variables whose failure rates vary with $l$ and $m$. Assume that $\lambda$ be the failure rate of a component, its value is given as follows.

$$
\lambda= \begin{cases}\lambda_{l} & m \leq 1, l \leq 3 \\ \lambda_{l+1} & m=2, l \leq 2 \\ \lambda_{3} & m=2, l=3 \\ \lambda_{3} & m=3, l \leq 3\end{cases}
$$

where $0<\lambda_{1}<\lambda_{2}<\lambda_{3}$. These assumptions coincide with engineer realities. When the number of failed components in a line $m \leq 1$, components can adjust failure rates according to the states of their "neighbours". When $m=2$, they can adjust not only according to the states of its three "neighbours", but also the number of failed components in another line. When the three components in a line fail, i.e., $m=3$, the failure rates of the components in another line will be $\lambda_{3}$, the highest one.

(3) The system and its components are repairable and the repair is assumed to be perfect. Each component is assumed to have its own dedicated repair crew. The repair-time durations of all components are exponential random variable with parameter $\mu(\mu>0)$ and are independent of the life-time durations.

If there are no other special explains, we will use "the system" to denote the 6-component lattice load-sharing Markov repairable system with spatial dependence defined above.

\subsection{States of the system}

We use $X(t)=\left(\begin{array}{lllll}X_{11}(t) & X_{12}(t) & X_{13}(t) ; \quad X_{21}(t) \quad X_{22}(t) \quad X_{23}(t)\end{array}\right) \quad(t \geq 0)$ to denote the state of the system, where $X_{i k}(t)=1$ or $0 \quad(i \in\{1,2\} ; k \in\{1,2,3\})$ according to when component $i k$ is functioning or failed. Since all the random variables involved in the system are exponentially distributed, $X(t)=\left(X_{11}(t) \quad X_{12}(t) \quad X_{13}(t) ; \quad X_{21}(t) \quad X_{22}(t) \quad X_{23}(t)\right)(t \geq 0)$ is a continuous-time Markov process, with state space $S=\{0,1\}^{6}$. Obviously, $2^{6}$ elements are included in $S$ and we call them the elementary states.

According to formulations of the system, the performance of each component depends on its spatial "neighbours" as well as the number of failed components in other lines. Hence, in general, the state space of the system can't be reduced by the number of failed components, although the components of the system are identical. However, taking account of symmetries of the lattice configuration $S$ can be reduced to $\tilde{S}$ with the twenty-four states. They are shown in Fig.1.

The definitions of state in $\tilde{S}$ are can be given in the following.

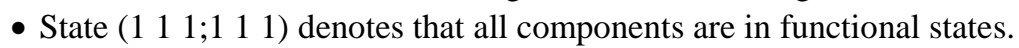

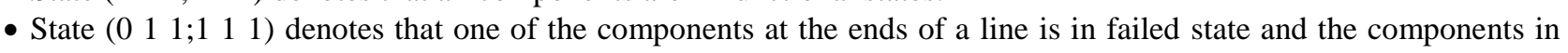
another line are functional. States $\left(\begin{array}{lllll}1 & 1 & 0 ; 1 & 1 & 1\end{array}\right),\left(\begin{array}{lllll}1 & 1 & 1 ; 0 & 1 & 1\end{array}\right)$ and $\left(\begin{array}{lllll}1 & 1 & 1 ; 1 & 1 & 0\end{array}\right)$ can be represented by $\left(\begin{array}{lllllll}0 & 1 & 1 ; 1 & 1 & 1\end{array}\right)$.

- State $\left(\begin{array}{llll}1 & 0 & 1 ; 1 & 1\end{array}\right)$ denotes that the component in the middle of one line is in failed state and the components in another

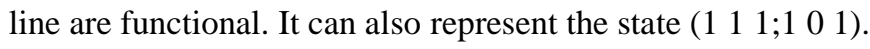

- State $\left(\begin{array}{llll}0 & 0 & 1 ; 1 & 1\end{array}\right)$ denotes that two adjacent components in a line are in failed states and the components in another

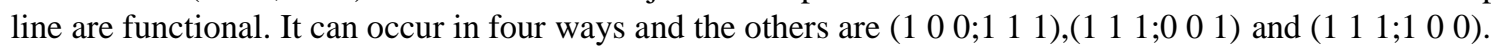

- State $\left(\begin{array}{llll}0 & 1 & 0 ; 1 & 1\end{array}\right)$ denotes that two components at two ends of the same line are in failed states and the components in another line are functional, it can also represent state (1 $111 ; 010)$.

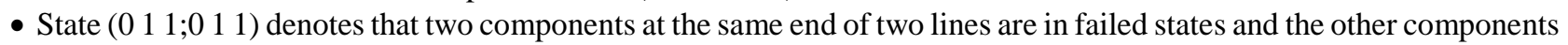
are functional. State $\left(\begin{array}{lllll}1 & 1 & 0 ; 1 & 1 & 0\end{array}\right)$ can also represented by it.

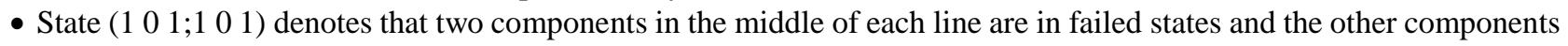
are functional.

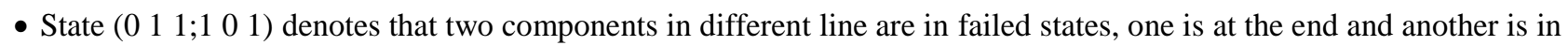

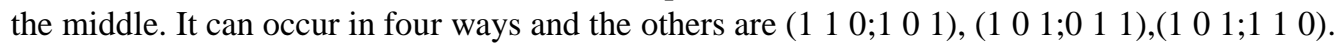

- State $\left(\begin{array}{llll}0 & 1 & 1 ; 1 & 1\end{array}\right)$ denotes that two components at different ends of two lines are in failed state and the other components

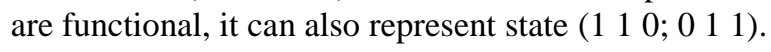

- State $\left(\begin{array}{llll}0 & 0 & 0 ; 1 & 1\end{array}\right)$ denotes that three components in a line are in failed states and the components in another line are functional. State $\left(\begin{array}{llll}1 & 1 & 1 ; 0 & 0\end{array}\right)$ can also be represented by it. 


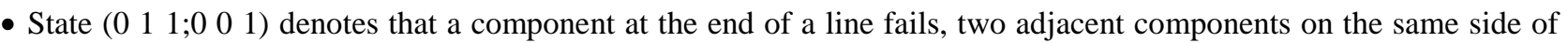
another line fail. It can occur in four ways and the others are $\left(\begin{array}{lllll}1 & 1 & 0 ; 1 & 0 & 0\end{array}\right),\left(\begin{array}{lllll}0 & 0 & 1 ; 0 & 1 & 1\end{array}\right)$ and $\left(\begin{array}{lllll}1 & 0 & 0 ; 1 & 1 & 0\end{array}\right)$.

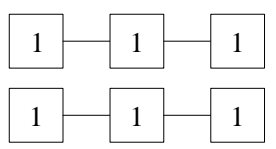

(1 $111 ; 111)$

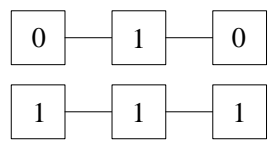

$\left(\begin{array}{lllll}0 & 1 & 0 ; 1 & 1 & 1\end{array}\right)$

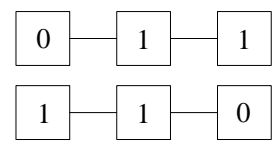

(0 11 1;1 110$)$

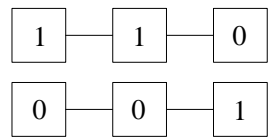

(1 $110 ; 0 ; 0001)$

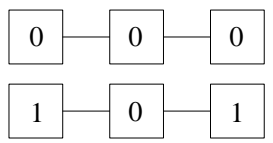

( $\left.\begin{array}{lllll}0 & 0 & 0 ; 1 & 0 & 1\end{array}\right)$

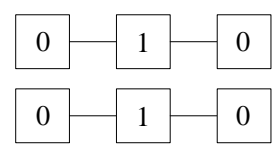

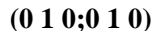

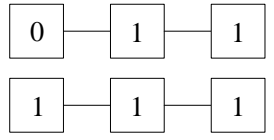

(0 1 1;1 11 1)

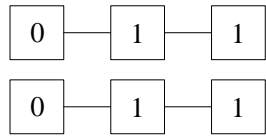

(0 1 1; 0111$)$

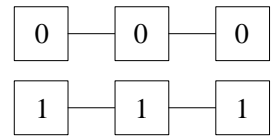

( 000 0; 1111$)$

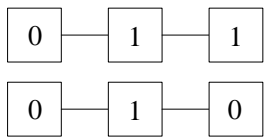

(0 10 0;0 10 )

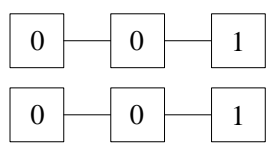

(l) 0 1; 000 1)

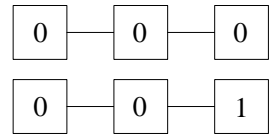

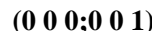

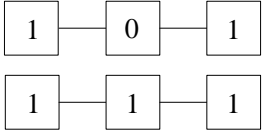

(1 0 0 1;1 111$)$

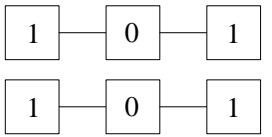

(1 0 1;1 0 1)

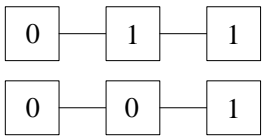

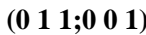

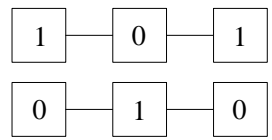

(1 $0101 ; 010)$

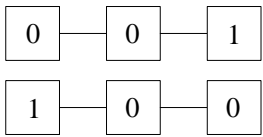

(0 0 1; 1000$)$

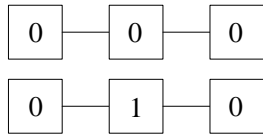

(0 00 0;0 10 0)

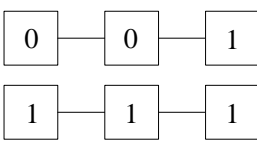

(0 $001 ; 11111)$

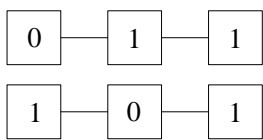

(0 1 1; 10 1)

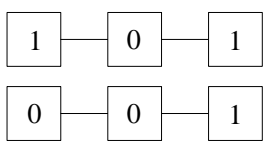

$(10$ 1;0 0 1)

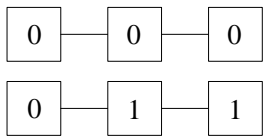

(0 0 0 0;0 11 1)

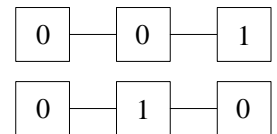

(0 0 1; 01010$)$

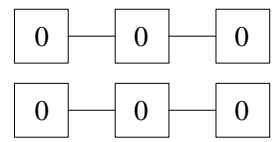

(0 0 0 0;0 000$)$

Figure 1: States of the 6 component lattice load-sharing system

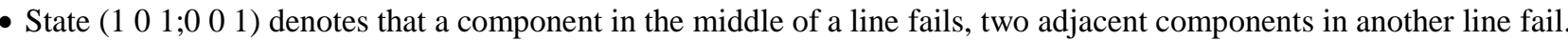

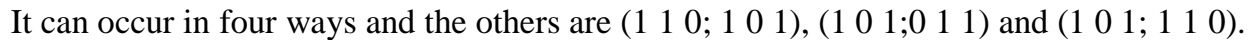

- State $\left(\begin{array}{llll}0 & 1 & 1 ; 1 & 1\end{array}\right)$ denotes that a component at the end of a line fails, two adjacent components on the different side of

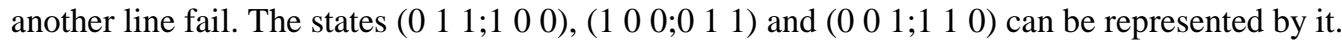

- State (1 $01 ; 010)$ denotes that a component in the middle of a line fails, two separated components in another line fail. State $\left(\begin{array}{lllll}0 & 1 & 0 ; 1 & 0 & 1\end{array}\right)$ can also be represented by it.

The other states can be defined by the symmetry of 0 and 1 . Let $\tilde{X}(t)$ be the vector-valued continuous-time Markov process with state space $\widetilde{S}$. We call it the aggregated process associated with $X(t)$ (see Ball et al.2002).

\section{State transition analysis of the system}

The evolution of the Markov system $\tilde{X}(t)$ is determined by the transitions among the twenty-four states. We will discuss the state transitions of the system in the following. Taking accounting of the numbers of failed components in a component's three "neighbours" and the number of failed components in another line, the state transitions of the system can be discussed. 
$\tilde{S}$ can be denoted as $\{1,2, \ldots, 24\}$ by numbering the states in Fig .2, from top to bottom, from left to right. Let $Q=\left[q_{i j}\right](i, j \in \tilde{S})$ be the transition rate matrix associated with $\tilde{X}(t)$.

Partition $\tilde{S}$ into $\tilde{S}_{1}=\{1,2, \ldots, 9\}, \tilde{S}_{2}=\{10,11, \ldots, 15\}, \widetilde{S}_{3}=\{16,17, \ldots, 21\}, \tilde{S}_{4}=\{22,23,24\}$ shown in Fig. 2 , then $Q$ can be represented as

$$
Q=\left(\begin{array}{llll}
Q_{11} & Q_{12} & Q_{13} & Q_{14} \\
Q_{21} & Q_{22} & Q_{23} & Q_{24} \\
Q_{31} & Q_{32} & Q_{33} & Q_{34} \\
Q_{41} & Q_{42} & Q_{43} & Q_{44}
\end{array}\right),
$$

where $Q_{i i}(i=1,2,3,4)$ is about transitions among states in $\tilde{S}_{i}, Q_{i j}$ is about transitions from $\tilde{S}_{i}$ to $\tilde{S}_{j}(i \neq j, i, j=1,2,3,4)$. From Fig. $2, Q_{i j}(i, j=1,2,3,4)$ can be given.

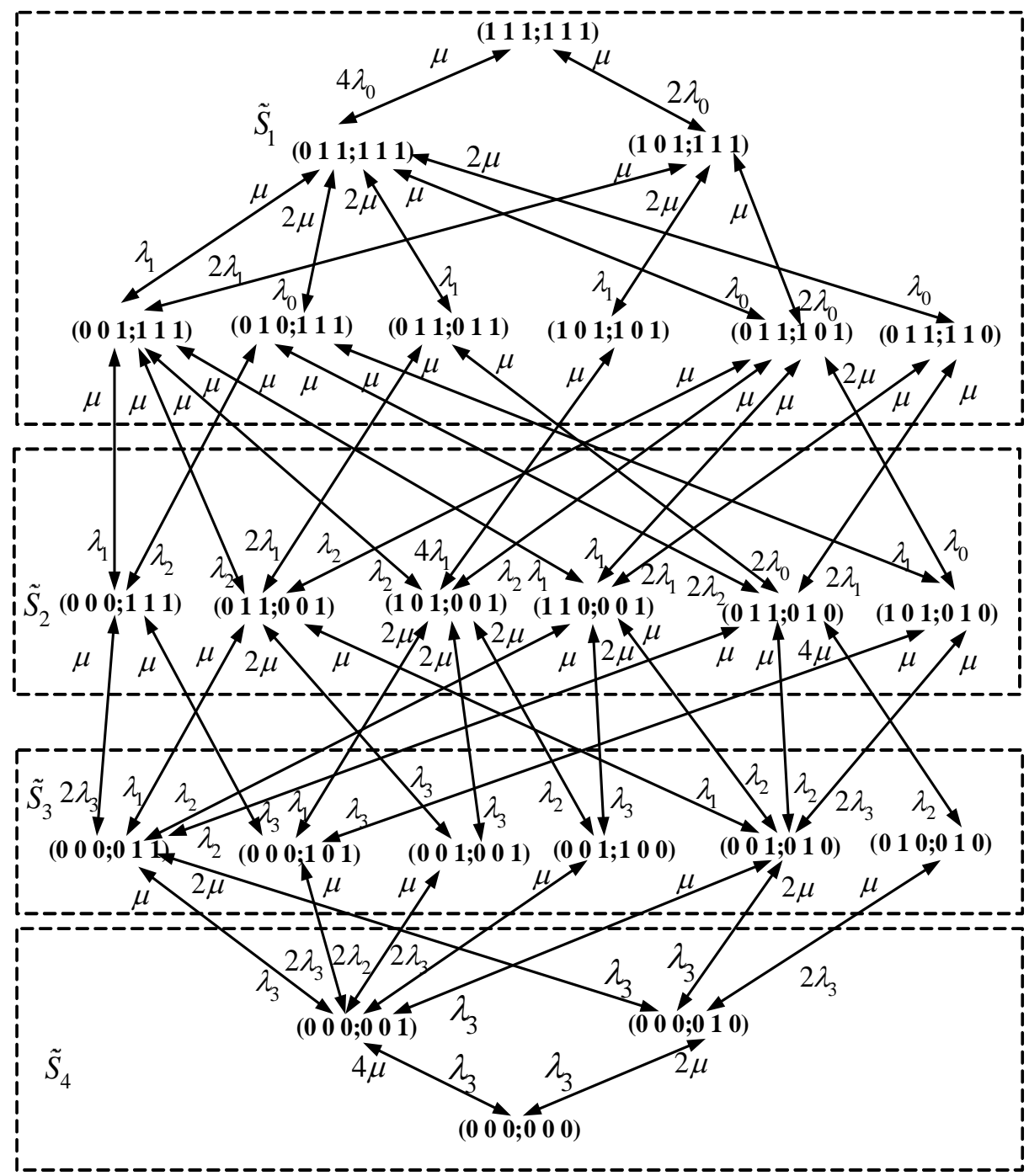

Figure 2: The state transition diagram of the system 


\section{Availability of the system}

Assume that $P(t)$ denote the distribution of the system at time $t$. Then $P(t)$ can be found from the Kolmogorov equations

$$
\frac{d P(t)}{d t}=P(t) Q
$$

given the initial law $P(0)=(1,0,0,0,0,0,0,0,0,0,0,0,0,0,0,0,0,0,0,0,0,0,0,0)$.

Using the initial condition $P(0)$ and taking Laplace transforms the equations reduce to (see Widder 1946)

$$
P^{*}(s)=P(0)(s I-Q)^{-1},
$$

where $P^{*}(s)$ is Laplace transform of $P(t)$.

Assume that the system output performance in the case that the number of functional components is more than two can satisfy the demand of customers. So, the acceptable states of the system are $\widetilde{S}_{1} \cup \widetilde{S}_{2} \cup \widetilde{S}_{3}$. The system's entrance to a state in the unacceptable set $\tilde{S}_{4}$ constitutes a failure (Lisnianski and Levitin 2003). Let $A(t)$ is the probability that the system is functioning at time $t$, i.e., the instantaneous availability of the system. Hence, its Laplace transform can be obtained by

$$
A^{*}(s)=P(0)(s I-Q)^{-1} \mu
$$

where $\mu=(1,1,1,1,1,1,1,1,1,1,1,1,1,1,1,1,1,1,1,1,1,0,0,0)^{T}$.Taking the inverse Laplace transform, we can get $A(t)$

Using $\pi$ to represent the stationary distributions of the system. It can be obtained by solving equations

$$
\left\{\begin{array}{l}
\pi Q=(0,0,0,0,0,0,0,0,0,0,0,0,0,0,0,0,0,0,0,0,0,0,0,0), \\
\pi e=1,
\end{array}\right.
$$

where $e$ is a 24-dimensional row vector and the elements are 1 . The asymptotic availability $A=\pi \mu$.

\section{A numerical example}

\subsection{Instantaneous availability}

Consider the 6-component lattice load-sharing Markov repairable systems with spatial dependence introduced in Section 2.1. Assume that the parameters of the system are $\lambda_{0}=0.2, \lambda_{1}=0.4, \lambda_{2}=0.8, \lambda_{3}=1.5$ and $\mu=0.8$. Substituting them into expressions in Sections 3 and 4 and taking Laplace and inverse Laplace transforms using Matlab, $A(t)$ can be given. Solving Equations (5), we can get $A=0.8772$.

In order to contrast, we consider the classical 6-component parallel Markov repairable systems. Assume that components of the system are identical and independent. Their life and repair times are respectively exponentially distributed with $\lambda_{0}=0.2$ and $\mu=0.8$. Define the state of the system as the number of failed components. It is easy to get the instantaneous availability $\bar{A}(t)$. The asymptotic availability $\bar{A}=0.9985$. The curves are shown in Fig. 3. The Fig. 3 indicates that the availability of the system with spatial dependence is lower than that of system with independent components.

\subsection{Probability of visiting to four state sets}

In the hierarchical structure of International Atomic Energy Agency (IANA), the safety performance indicators (SPIs) evaluation is based on two regions for indicator values, with the help of aggregation. They are satisfactory (acceptable) and unsatisfactory (unacceptable or failed) regions. The satisfactory regions are divided into three zones: secure, degraded and warning (caution) (see Saqib and Siddiqi 2008 and Wang and Cui 2013). 


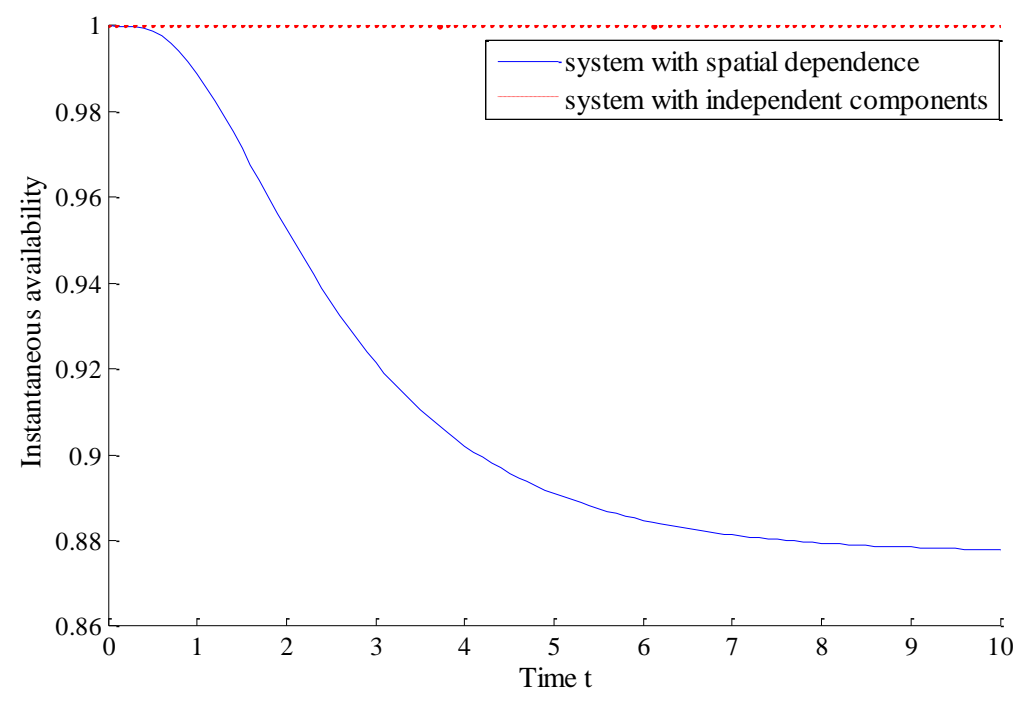

Figure 3: Instantaneous availability of the two 6-component systems

According to the number of failed components, we also divide the 24 states of the system with spatial dependence into the four categories, $\tilde{S}_{1}, \tilde{S}_{2}, \tilde{S}_{3}$ and $\tilde{S}_{4}$. They are described as follows. In the secure state set $\tilde{S}_{1}$, the number of failed components in the system is not more 2 . In the degraded state $\tilde{S}_{2}$, the number of failed components is 3 . In the warning state set $\tilde{S}_{3}$, the number of failed components in the system is 4 . In the failed state set $\tilde{S}_{4}$, the number of failed components in the system is 5 or 6 .

Using (4), we can obtain the Laplace transform of the instantaneous probability of the system in the secure, degraded, warning and failed state sets

$$
\begin{gathered}
S^{*}(s)=P(0)(s I-Q)^{-1} \mu_{1}, \\
D^{*}(s)=P(0)(s I-Q)^{-1} \mu_{2}, \\
W^{*}(s)=P(0)(s I-Q)^{-1} \mu_{3}, \\
F^{*}(s)=P(0)(s I-Q)^{-1} \mu_{4},
\end{gathered}
$$

where

$$
\begin{gathered}
\mu_{1}=(1,1,1,1,1,1,1,1,1,0,0,0,0,0,0,0,0,0,0,0,0,0,0,0)^{T}, \\
\mu_{2}=(0,0,0,0,0,0,0,0,0,1,1,1,1,1,1,0,0,0,0,0,0,0,0,0)^{T}, \\
\mu_{3}=(0,0,0,0,0,0,0,0,0,0,0,0,0,0,0,1,1,1,1,1,1,0,0,0)^{T} \\
\mu_{4}=(0,0,0,0,0,0,0,0,0,0,0,0,0,0,0,0,0,0,0,0,0,1,1,1)^{T} .
\end{gathered}
$$

After taking Laplace inverse transformation, we can get the instantaneous probability of the system in the secure, degraded state set, warning and failed state sets. The curves are shown in Fig. 4. The asymptotic probability $S=\pi \mu_{1}=0.5687, D=\pi \mu_{2}=0.1648, W=\pi \mu_{3}=0.1436$ and $F=\pi \mu_{4}=0.1229$.

\section{Conclusions}

The lattice load-sharing Markov repairable systems with spatial dependence discussed in this paper extends the classical Markov repairable system to one whose components are arranged in a lattice and the failure rates of all components vary with the number of failed components in their "neighbours" and the performance situation of 


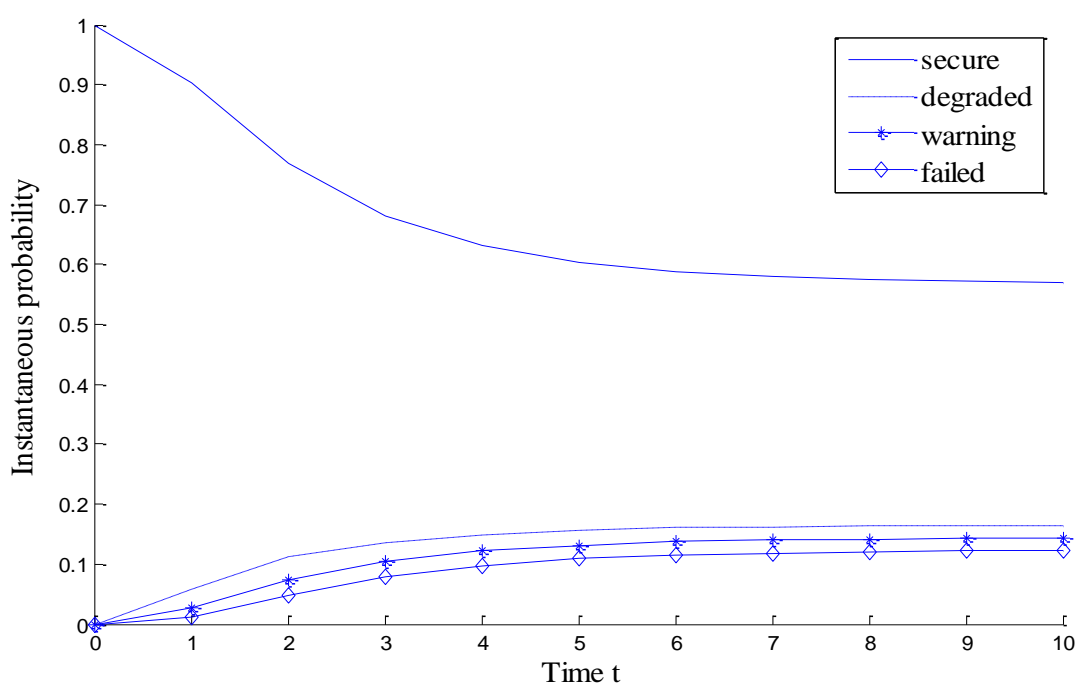

Figure 4: Instantaneous probabilities of visiting to four state sets

components in the other line. Availability, probabilities of visit to secure, degraded, warning and failed state sets of the system are considered. The work might provide basis for the reliability analysis of load-sharing systems with two-dimensional spatial pattern.

We started with a relatively simple model and more complex and realistic models could be built in the future. General $n \times m$ lattice load sharing systems with spatial dependence, Semi-Markovian systems with spatial dependence, systems with other spatial patterns, such as a rosette, and three-dimensional patterns, are worth being considered.

\section{Acknowledgements}

We thank editors and referees for their detailed comments and suggestions. This work is supported partly by the NSF of China Grants (71201111, 11471218) and Hebei Province (A2015210103).

\section{References}

1. W. Kuo and M.J. Zuo, "Optimal reliability modelling”, Wiley, New York, 2003.

2. K.C. Kapur and L.R. Lamberson, "Reliability in engineering design", Wiley, New York, 1977.

3. R.K. Iyer and D.P. Rossetti, "A measurement-based model for workload dependency of CPU errors", IEEE Transactions on Computer; vol.35, no.6, pp. 511-519, 1986.

4. A. Barros, C. Berenguer and A. Grall, "Optimization of replacement times using imperfect monitoring information", IEEE Transactions on Reliability, vol.52, no.4, pp. 523-533, 2003.

5. S.V. Amari, K.B, Krishna, H. Pham, "Tampered failure fate load-sharing systems: status and perspectives". Handbook of Performability Engineering, pp. 291-308, 2008.

6. M. Jain, R. Gupta, "Load sharing M-out of-N: G system with non-identical components subject to common cause failure", Int. J. Mathematics in Operational Research, vol.4, no.5, pp. 586-605, 2012.

7. L.Y. Wang, X.J. Jia and J. Zhang, "Reliability evaluation for Multi-State Markov repairable systems with redundant dependencies", Quality Technology and Quantitative Management, vol.10, no.3, pp. 277-289, 2013.

8. H.Y. Yu, C.B. Chu, E. Chatelet and F Yalaoui, "Reliability optimization of a redundant system with failure dependencies", Reliability Engineering and System Safety, vol.92, no.12, pp. 1627-1634, 2007.

9. G. Levitin and L.D. Xing, "Reliability and performance of multi-state systems with propagated failures having selective effect", Reliability Engineering and System Safety, vol. 95, no. 6, pp. 655-661, 2010.

10. G. Maaroufi, A. Chelbi and N. Rezg, "Optimal selective renewal policy for systems subject to propagated failures with global effect and failure isolation phenomena", Reliability Engineering and System Safety, vol. 114, no. 6, pp. 61-70, 2013.

11. L.Y. Wang and S.B. Si, "Reliability analysis of circular Markov repairable systems with spatial dependence", Journal of northwestern polytechnical university, vol. 32, no. 6, pp. 923-928, 2014.

12. F. Ball, R.K. Milne and G.F. Yeo, "Multivariable semi-Markov analysis of burst properties of multi-conductance single ion channels", Journal of Applied Probability, vol. 39, no. 1, pp. 179-196, 2002. 
13. A. Lisnianski and G. Levitin, "Multi-state system reliability, assessment, optimization and application", Singapore: World Scientific Publishing Co. Pte. Ltd., 2003.

14. D.V. Widder, “The Laplace Transform”, Princeton University Press, 1946.

15. N. Saqib, and M.T. Siddiqi, "Aggregation of safety performance indicators to higher-level indicators", Reliability Engineering and System Safety, vol. 93, no. 2, pp. 307-315, 2008.

16. L.Y. Wang and L.R. Cui, "Performance evaluation of aggregated Markov repairable systems with multi-operating levels", AsiaPacific Journal of Operational Research, vol. 30, no. 4, pp. 1350003-1-27, 2013.

Liying Wang is Professor of Department of Mathematics \& Physics at Shijiazhuang Tiedao Institute, China.

Yuran Tian and Zhaona Pei are graduate students of Department of Mathematics \& Physics at Shijiazhuang Tiedao Institute, China. 